INRA Prod. Anim.,

2014, 27 (1), 31-40

\title{
Particularités pathologiques des ruminants domestiques en estive dans les montagnes françaises
}

\author{
J.-P. ALZIEU', J. BRUGÈRE-PICOUX', C. BRARD \\ ${ }^{1}$ Laboratiore Vétérinaire Départemental de l'Ariège, F-09008 Foix, France \\ ${ }^{2}$ Ecole Nationale Vétérinaire d'Alfort, F-91700 Maisons-Alfort, France \\ ${ }^{3}$ Clinique Vétérinaire du Piémont, F-64800 Mirepeix, France \\ Courriel : jpalzieu@cg09.fr
}

La transhumance en montagne, très développée en France, revêt pour les éleveurs de ruminants un intérêt agronomique évident. Cependant, l'impact médical et zootechnique de diverses pathologies, pour la plupart déjà portées par leurs hôtes ruminants avant la montée en estive peut s'avérer un facteur limitant à sa bonne valorisation.

L'élevage en montagne est pratiqué dans de nombreuses zones en France : deux sont très significatives, d'une part, avec les Massifs pyrénéen et alpin, et d'autre part, le Massif Central moins accidenté et de basse et moyenne altitude. Les principales espèces domestiques qui y sont élevées sont les bovins et les ovins de type allaitant, des génisses et vaches laitières dans le Nord du Massif alpin et accessoirement les équins.

L'élevage et les pathologies rencontrés durant la phase hivernale de stabulation ne diffèrent guère de ceux rencontrés chez les éleveurs sédentaires : ils ne diffèrent que par la durée allongée d'occupation des bâtiments d'élevage avant la sortie à l'herbe. Ils ne seront donc pas évoqués ici.

L'objectif de cet article est de décrire les particularités pathologiques des ruminants lors de l'estive. Ainsi, nous traiterons des dominantes pathologiques des bovins et ovins. Toutefois, il convient d'évoquer aussi les pathologies induites dans certaines zones par les prédateurs comme le loup ou l'ours avec leurs cortèges de morbidité et de mortalité, devenues pour les éleveurs un facteur limitant de la transhumance.

\section{1 / Le contexte de l'élevage en montagne}

\section{1 / L'intérêt de l'utilisation des estives}

Sous le terme générique « d'estives » (ou encore « alpages ») est englobée la majeure partie des pâturages et parcours, souvent de grandes étendues disponibles pour le pâturage des bovins, ovins ou encore équins. La valeur alimentaire et la masse de la ressource fourragère des estives sont connues depuis longtemps par les éleveurs : cet élément est décisif dans le système d'élevage avec transhumance. Cela permet de décharger l'exploitation de base, de bon nombre d'animaux sub-adultes et adultes pendant les 4 (à 5) mois d'estive : les éleveurs sont ainsi libres pour assurer la fauche de leurs prairies, optimiser la récolte de fourrages, pour curer et désinfecter les étables et réaliser un réel vide sanitaire. Cette pratique autorise aussi d'élever beaucoup plus d'animaux que dans un élevage sédentaire : dans le cadre d'une politique d'élevage subventionnée liée aux nombres/quotas de production, l'impact économique est majeur ; de plus, un soutien financier particulier pour l'élevage en montagne est permis grâce aux primes à l'herbe et de handicap naturel.

\section{2 / Les inconvénients de l'éleva- ge en montagne}

Ils sont multiples mais malgré tout, moindres que les avantages précités : de ce fait, l'élevage en montagne se maintient bien avec cependant plusieurs bémols, liés à certains contextes sanitaires et par secteurs, aux super-prédateurs (ours, loup...).

\section{a) Inconvénients liés à l'étendue des estives}

Les pâtures d'estives couvrent usuellement de très grandes surfaces (de 1000 à 10000 ha) : les bovins et les ovins y sont conduits généralement de façon très extensive, avec une répartition spatiale très élargie sur le terrain ; la surveillance des animaux en est rendue plus difficile. $\mathrm{Vu}$ cette dispersion, la détection des malades s'en trouve souvent retardée et par conséquent le traitement de ceux-ci. Pourtant, de la précocité du diagnostic dépend très souvent la réussite du traitement ou de la méta-phylaxie à instaurer. Enfin et surtout, malgré de nombreux efforts collectifs d'équipement en parcs de contention, la dispersion des animaux sur le terrain (habituellement, un à deux pour 1000 hectares) impose un délai pour leur regroupement et les interventions prévues.

\section{b) Inconvénients sanitaires liés au cheptel} collectif

Les cheptels d'estive sont, en très grande majorité, des cheptels collectifs regroupant des troupeaux de provenance diverses (éleveurs locaux « ayants-droit», éleveurs "invités ») : chaque cheptel dispose de fait d'un statut sanitaire qui lui est propre à l'entrée en estive. Cette diversité vis à vis des maladies infectieuses (hors maladies officiellement réglementées) et parasitaires constitue le problème majeur dans la gestion sanitaire de l'estive (les responsables d'estive ont d'ailleurs souvent beaucoup de mal à faire adopter un règlement sanitaire d'estive, optimal, commun à tous).

De ce fait, en fonction des différentes préventions réalisées avant estive (vaccinations, vermifugations...), une grande variation de la nature des pathologies est observée. Les sujets naïfs de première 
Figure 1. Prévalence très élevée de l'IBR en zone de transhumance dans la région Midi-Pyrénées.

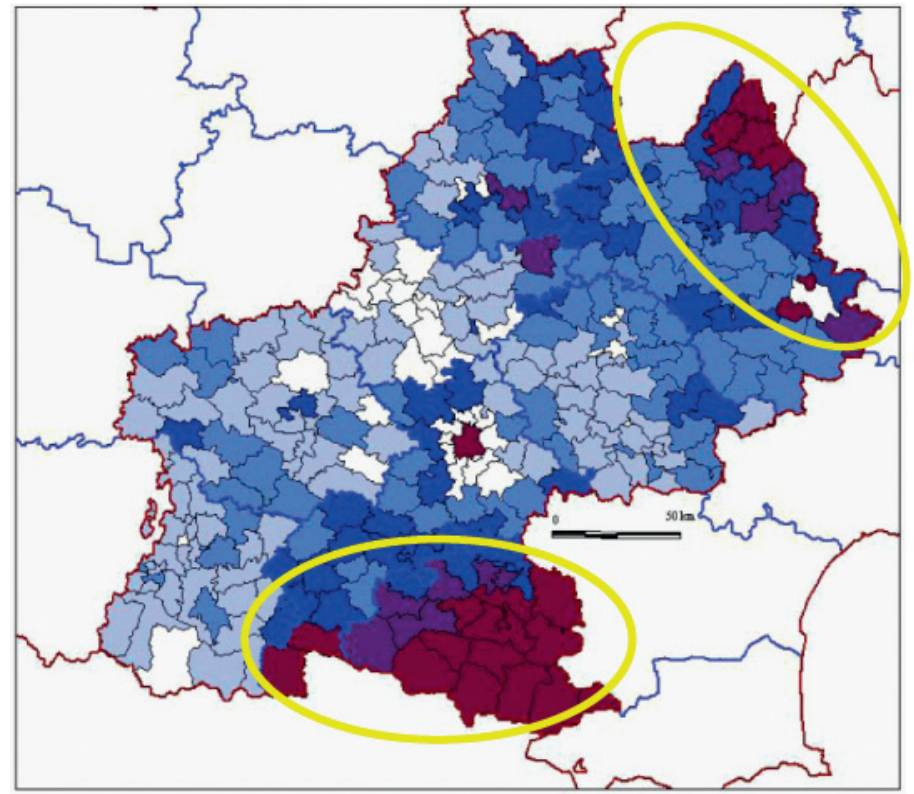

saison de pâture ou les moins prémunis constituent le réservoir infectieux d'agents pathogènes, sources fréquentes d'épizooties (maladies respiratoires des veaux), mais le plus souvent générateurs de contaminations insidieuses sub-cliniques (infectieuses, gale, parasitisme interne...).

\section{c) L'influence du système d'élevage}

Les pathologies d'estive sont fortement influencées par les catégories d'animaux transhumants : en particulier, la présence des jeunes de l'année (globalement plus réceptifs et plus sensibles) avec leurs mères accroît les risques de survenue de certaines pathologies. Il en est ainsi des troubles respiratoires infectieux d'allure épizootique chez les bovins, très fréquents dans les Pyrénées et le Massif Central, mais en revanche, peu rencontrés dans les Alpes où les éleveurs envoient plutôt en estive le cheptel de renouvellement de $2^{\text {ème }}$ saison de pâture et des adultes (cheptel à prédominance laitière dans le Nord des Alpes). De même, le parasitisme interne affecte fortement les bovins jeunes et les agneaux/agnelles non encore immunisés ; les parasites sont donc fortement recyclés par ceux-ci sur les pâtures des estives.

\section{d) Le cas particulier de l'IBR}

La rhinotrachéite infectieuse bovine (IBR), due à l'herpès-virus $\mathrm{BoHV}_{1}$ est actuellement objet de réglementation, en vue de son éradication (Memeteau 2011). Nombreux sont les cheptels bovins transhumants infectés : malgré la vaccination stratégique préconisée, force est de constater le maintien fréquent de la circulation virale en estive, liée à la problématique des cheptels collectifs et au manque de rigueur dans la stratégie vacci(photo J.P. Alzieu).

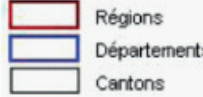

Prévalence Cheptel

Plus de $50 \%$ De $35 \%$ à $50 \%$ De $20 \%$ a $35 \%$ de $10 \%$ \& $20 \%$ Pas de positif

très souvent sous-estimés liés à la prédation directe " officiellement reconnue » dans les troupeaux bovins, ovins et équins (en particulier dans les Pyrénées ariègeoises où les ours se concentrent, figure 2). En revanche, sont toujours passées sous silence, les pertes fréquentes liées à la fuite et au stress se traduisant par les avortements précoces chez les ovins ou les morts très suspectes par chutes dans des endroits improbables et les soucis liés à une dispersion invraisemblable des ovins en montagne. La productivité numérique en élevage ovin s'effondre alors à $0,5 \mathrm{ou}$ 0,6 agneau/an (au lieu des 0,7 officiellement requis pour l'accès aux primes d'élevage) (source : DDT de l'Ariège). Devant de tels problèmes, plusieurs estives - principalement ovines- ont été désertées et de nombreux éleveurs démotivés ont préféré, au mieux, s'exiler en d'autres lieux, au pire arrêter leur activité.

Il en est de même avec le loup chassant en meute, pratiquant savamment le rabat des ovins et autres équins vers des falaises. Une cause différente, mais avec les mêmes effets que l'ours sur les animaux et les mêmes conséquences désastreuses pour l'économie montagnarde (en particulier, dans la partie centrale et méridionale des Alpes). Les témoignages et les constats de terrain sont accablants sur ces actes de prédation, considérés par les éleveurs comme sous-évalués par les pouvoirs publics.

Il ne saurait ici être question d'éluder le rôle néfaste des super-prédateurs que sont l'ours (Massif pyrénéen) et le loup (Massif alpin essentiellement) sur les productions animales en estive.

La réintroduction d'ours slovènes dans les Pyrénées a été responsable de dégâts

\section{f) Le risque lié au réservoir sauvage d'agents pathogènes pour l'animal et l'Homme}

Le risque lié au réservoir sauvage d'agents pathogènes a fait l'objet d'une conférence commune des académies

Figure 2. Les dégâts dus à l'ours en Ariège ont conduit à l'euthanasie de ce bélier de race tarasconnaise inscrit, fruit de la patiente sélection des éleveurs locaux

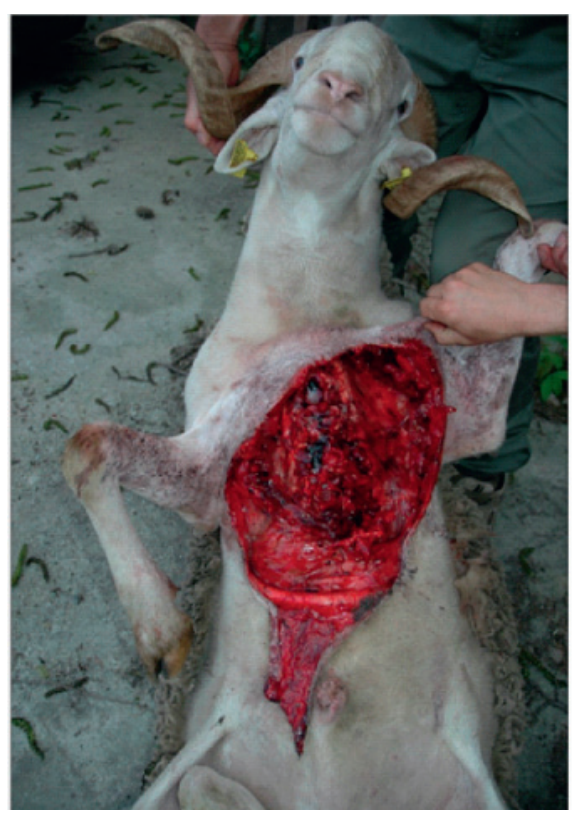


vétérinaire et d'agriculture de France le 9 novembre $2011^{1}$. En montagne, nous citerons plus particulièrement la réapparition relativement récente de la brucellose (à Brucella melitensis) en avril 2012, à la suite d'une contamination humaine par du reblochon fabriqué au GrandBornand en Haute-Savoie. Cette résurgence dans un cheptel bovin a justifié la recherche de l'origine de cette contamination au sein des espèces de ruminants sauvages. L'infection a ainsi été démontrée en Haute-Savoie chez des bouquetins (10 positifs sur 22 prélèvements soit $45 \%$ d'animaux infectés) alors qu'une seule femelle chamois s'est révélée positive.

Ceci témoigne que l'éradication de la brucellose obtenue en France après les grandes prophylaxies des dernières décennies peut être mise en défaut par la présence d'espèces sauvages ne présentant pas les garanties nécessaires pour la protection de nos cheptels domestiques. Il reste à connaître l'origine exacte de ces bouquetins qui ont pu rester infectés pendant plusieurs années avant de contaminer un cheptel bovin dans un département montagnard indemne depuis 1999. Il est aussi inquiétant de découvrir à cette occasion que l'Homme est devenu une " espèce sentinelle » pour la brucellose !

\section{2 / Dominantes pathologiques dans l'espèce bovine}

Elles peuvent être classées par ordre de fréquence et/ou d'importance relative dans l'ordre suivant : les maladies respiratoires infectieuses, les boiteries et les troubles digestifs (trio constant dans tous les modes d'élevage), suivis selon les contextes épidémiologiques par la babésiose, la besnoitiose et le parasitisme général clinique et sub-clinique (Collectif Réseau VEGA 1993b et e) (tableau 1).

\section{1 / Les maladies respiratoires infectieuses}

Souvent appelée « grippe » par les éleveurs, ce syndrome respiratoire est la résultante complexe de facteurs physiques favorisants liés à l'environnement et de facteurs déterminants infectieux viraux et bactériens.

\section{a) De puissants facteurs favorisants d'environnement}

Outre les facteurs favorisants spécifiques («petits » poumons de faible capacité chez les ruminants, défense des muqueuses respiratoires d'autant plus faible que l'animal est jeune...), les facteurs d'environnement (écarts thermiques jour/nuit conséquents en montagne, conditions climatiques très changeantes) influent hautement sur les mécanismes de défense naturels des muqueuses trachéale et nasale.

\section{b) L'association " de malfaiteurs " des} virus et bactéries

Les facteurs déterminants restent les agents infectieux viraux et bactériens. Les virus sont les agents initiateurs avec, par ordre de pathogénicité et de fréquence, le RSV (virus syncytial respiratoire), le $\mathrm{PI}_{3}$ (Para-influenza 3), l'herpes-virus $\mathrm{BoHV}_{1}$ de l'IBR et le BVDV (virus de la maladie des muqueuses). Les bactéries jouent le rôle de surinfection et d'amplification, avec l'atteinte extensive du parenchyme pulmonaire : parmi cellesci, Mannheimia haemolytica joue le rôle principal.

Ce contexte infectieux est lourdement amplifié par le mélange de cheptels de statuts sanitaires différents et la présence de bovins jeunes réceptifs et très sensibles. La chronologie clinique évolue classiquement de la forme fébrile initiale d'invasion, vers la forme subaigüe (toux, anorexie persistante) pouvant elle-même conduire à la forme chronique avec toux persistante, retards de croissance, et amaigrissement conduisant très souvent à l'état de non-valeur économique.

\section{c) Une évolution de type épizootique}

L'allure la plus fréquente en estive est épizootique, évoluant classiquement sur deux à trois semaines. Aussi, les bovins jeunes de première saison de pâture (veaux non sevrés en élevage allaitant) paient-ils un lourd tribut à ces maladies infectieuses, notamment dans les Pyrénées ou le Massif Central où ce type d'élevage est très prévalent.

En l'absence de prévention vaccinale des jeunes veaux nés durant l'hiver, avant la montée en estive, la morbidité observée est souvent très élevée $(>50 \%)$ et la mortalité est fréquente avec de grandes variations (classiquement de l'ordre de 5 à $10 \%$ ) selon la précocité du diagnostic et du traitement. A un degré moindre, les vaches primipares, ou encore les génisses n'ayant jamais fréquenté antérieurement les alpages (système d'élevage usuel dans les Alpes) peuvent présenter une pathologie de ce type.

d) La surveillance et la précocité du diagnostic, clés de la réussite

De la précocité du diagnostic (plus délicat en montagne du fait du nombre et de la dispersion des bovins sur les pâturages et de la qualité de la surveillance) dépend la réussite du traitement. $\mathrm{La}$ stratégie habituelle est le traitement individuel dès les premiers cas, suivie très rapidement du traitement anti-infectieux dit « méta-phylactique », dès que la morbidité atteint le seuil de 10\% : cela s'avère nécessaire pour prendre «de vitesse » la maladie et réduire la pression infectieuse générale. Ces épizooties sont très coûteuses tant en temps consacré aux

Tableau 1. Les dominantes pathologiques dans l'espèce bovine (par ordre décroissant de fréquence et/ou d'importance).

\begin{tabular}{|l|c|}
\hline Entité pathologique & Importance relative \\
\hline 1. Les maladies respiratoires infectieuses & +++ \\
\hline 2. Les boiteries & $++(+)$ \\
\hline $\begin{array}{l}\text { 3. Les troubles digestifs } \\
\text { - liés à l'herbage }\end{array}$ & $+\mathbf{+}(+)$ \\
\hline 4. La babésiose & $+(+)$ \\
\hline 5. La besnoitiose & $+(++)$ \\
\hline 6. Le parasitisme interne et externe & $++(+)$ \\
\hline
\end{tabular}

Légende : + (faible) à ++++ (très élevé), entre parenthèses, les variations possibles.

\footnotetext{
${ }^{1}$ Communications disponibles sur le site «http ://académie-agriculture.fr »
} 
soins qu'en frais liés aux anti-infectieux et anti-inflammatoires utilisés.

\section{e) Une prévention possible mais encore trop peu appliquée}

Compte-tenu du fait qu'il n'existe aucune possibilité d'action sur les facteurs d'environnement, la seule prévention réside en la prévention médicale par la vaccination, principalement des veaux, avant la montée en estive, a minima contre les virus RSV et BVD et si possible contre les bactéries Pasteurella $s p$ et Mannheimia haemolytica. Malheureusement encore, de trop nombreux éleveurs n'appliquent pas cette prévention, malgré la très large communication des vétérinaires et des organismes à vocation sanitaire sur ce sujet.

\section{2 / Les boiteries}

Les boiteries constituent le quotidien des éleveurs en estive avec deux composantes principales, le panaris interdigité et l'entité appelée « Mal de l'herbe », assimilable à une fourbure (Collectif Réseau VEGA, 1993 a et e).

\section{a) Le phlegmon inter-digité ou "panaris} inter-digité »

Bien connu et ubiquitaire, le phlegmon inter-digité (figure 3 ) résulte d'une infection nécrosante des tissus mous interdigitaux, par le bacille de la nécrose, Fusobacterium necrophorum. Les blessures au pied mais surtout l'humidité et la boue (mélange de fèces) autour des points d'eau et des parcs de contention sont des facteurs hautement favorisants. La boiterie pyrétique générée est subite et franche, et siège généralement sur un

Figure 3. En estive, les affections podales telles que ce phlegmon inter-digité varient du cas isolé à des successions de cas d'allure pseudo-épizootique (photo J.P. Alzieu).

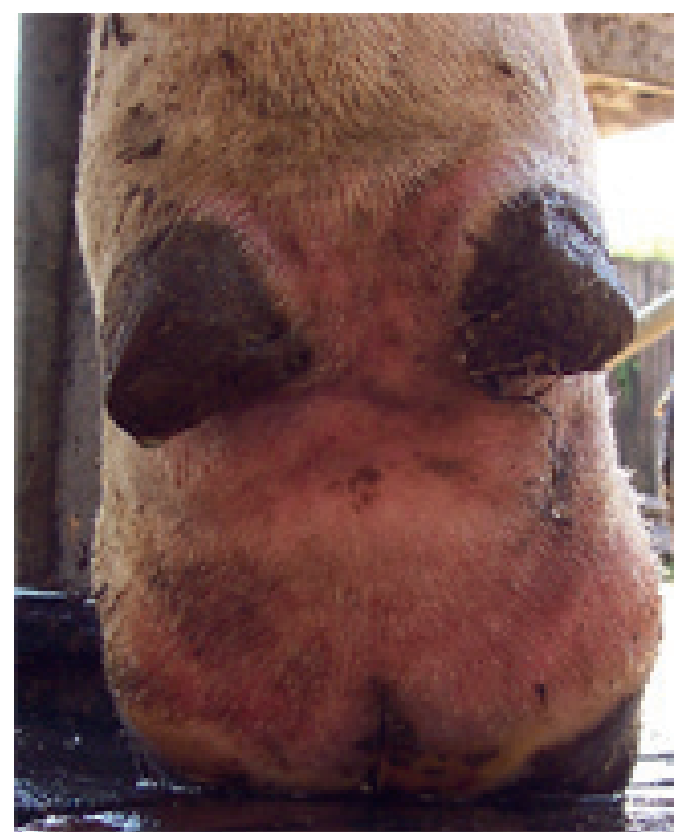

Le pronostic est toujours grave, lié à la souffrance intense et aux fréquentes surinfections bactériennes locales. Même si le traitement (anti-infectieux, antiinflammatoires) est précoce, il s'avère souvent nécessaire de redescendre le(s) bovin(s) atteint(s) de l'estive pour pouvoir assurer les traitements adéquats. La prévention réside à n'envoyer en estive que d'animaux habitués à ces pâturages, le plus souvent « adaptés » dès leur première année de vie (veaux, agnelles).

\section{3 / Les troubles digestifs et assimilés}

Ils sont principalement liés à la richesse de l'herbage, au contexte pédologique et peuvent induire des pathologies parfois graves telles les entérotoxémies, les charbons symptomatiques ou encore la coccidiose.

\section{a) Les diarrhées « banales »}

En relation avec la richesse en eau, et en azote soluble de l'herbe de printemps, la dysbiose de la flore intestinale provoque une diarrhée fréquente, transitoire, d'intensité variable, souillant le périnée et les faces internes des cuisses (« signes de l'écusson ») et de prévalence élevée, souvent supérieure à $50 \%$. Ses conséquences sont bénignes et généralement n'obligent pas à l'administration de pansements digestifs et d'anti-infectieux. En revanche, ces diarrhées constituent par contre un facteur très hautement favorisant pour l'expression de la coccidiose chez les veaux.

\section{b) La coccidiose}

Même si certains éleveurs persistent à distinguer sur les veaux de l'année, coccidiose d'étable et coccidiose de pâture, force est de constater que l'origine de l'infection se produit en étable et que les facteurs favorisants de l'estive (herbage, stress divers) permettent son expression (Alzieu et Chartier 2007). Seules les espèces Eimeria bovis et Eimeria zuernii sont réputées pathogènes (Alzieu et Le Goupil 2006, Alzieu et Chartier 2007) : elles induisent une diarrhée caractéristique (fèces brunes plus ou moins mêlées de sang) en relation avec une recto-colite intense : les cas en estive sont sporadiques (de 1 à $3 \%$ ).

En estive, le diagnostic de la coccidiose est souvent tardif, le début d'évolution étant peu caractéristique laissant présager une diarrhée banale. Ce n'est qu'au stade avancé d'épreintes et ténesmes, souvent associé à de la déshydratation, que le traitement est instauré (sulfamidothérapie en injection, acétonitriles de benzène per os), avec un succès thérapeutique très variable. Ce stade avancé fait toute la gravité de la coccidiose en 
estive. La prévention réside, en phase de stabulation, en la gestion médicale rationnelle de la coccidiose dans les deux premiers mois de vie du veau. L'administration méta-phylactique stratégique d'anticoccidien, généralement une semaine avant l'âge d'apparition habituelle des signes cliniques, autorise le développement d'une réponse immunitaire adaptative pérenne, à composante cellulaire.

\section{c) Les maladies à germes anaérobies}

Qu'elles soient d'origine digestive (entérotoxémies à Clostridium perfringens) ou pédologiques (charbon bactéridien à Bacillus anthracis ou charbons symptomatiques dus à Clostridium septicum, Clostridium novyi, Clostridium sordelli...), ces maladies sont souvent favorisées par divers stress d'ordre alimentaire (richesse de l'herbe de printemps) ou physiques (facteurs d'environnement, regroupements....). La prévention, seule solution possible, réside en la vaccination spécifique avant l'estive, fréquemment réalisée par les éleveurs (souvent requise par les règlements sanitaires internes des estives).

\section{4 / La babésiose (ou piroplasmose)}

La babésiose due principalement chez les bovins à Babesia divergens, génère un syndrome hémolytique grave avec urines foncées et mousseuses et associé à de la diarrhée en " corde » (Devos et Geysen 2005a, L'Hostis et al 2011, figure 4).

Il s'agit d'une maladie saisonnière survenant principalement en estive en mai-juin et accessoirement (la plupart des infections sont asymptomatiques) en septembre-octobre, rencontrées dans tous les massifs montagneux. Les zones de sous-bois de feuillus et de landes de basse et moyenne montagne (étage de 800 à 1300 mètres d'altitude) sont les seules zones réellement favorables à l'évolution de la tique tri-phasique Ixodes ricinus, principal vecteur du protozoaire (Devos et Geysen 2005a et b). Les cas ne surviennent que dans certaines zones à risque (souvent clairement connues par les éleveurs) principalement chez les bovins âgés de plus de 2 ans. La babésiose clinique aigüe reste cependant peu fréquente, de l'ordre de 0,4 à $1 \%$ des animaux (Devos et Geysen 2005b, L'Hostis et al 2007) dans les zones à risque ; cependant, à la faveur de modifications (reconstitution de cheptel avec des sujets naïfs, occupation de nouvelles zones de pâturages), des successions de cas, d'allure épizootique, peuvent survenir.

Pour des raisons d'effectifs à surveiller et de d'éparpillement relatif des bovins sur les pâtures, le diagnostic est souvent tardif : le bovin est alors très anémié

Figure 4. La diarrhée "en corde " ainsi que les urines foncées et mousseuses sont caractéristiques de la babésiose (photos G. Levasseur).

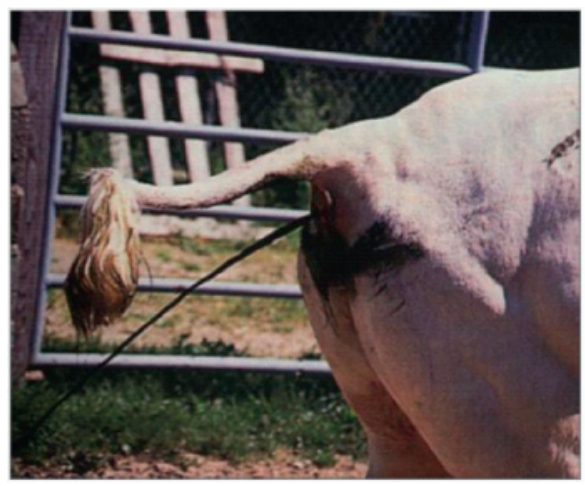

voire en subictère (hématocrite et numération erythocytaire effondrés). Le traitement s'avère souvent décevant avec la mort fréquente du bovin (Chauvin et al 2007). La seule prévention réside en l'administration préventive d'imidocarbe (une semaine avant l'arrivée dans la zone à risque), conférant une chimio-protection d'environ 5 semaines. L'idéal reste de laisser les bovins jeunes âgés de moins de 1 an (réceptifs mais non sensibles) s'infecter dans les zones à risque, conduisant à leur immunité (état de stabilité endémique) : ainsi, les bovins jeunes ayant fréquenté précocement les zones contaminantes revêtent un intérêt majeur pour leur carrière ultérieure en estive.

\section{5 / La besnoitiose (ou « anasar- que " des bovins)}

La besnoitiose bovine, ou « anasarque des bovins », due au protozoaire Besnoitia besnoiti, endémique en Europe du Sud (Pyrénées, France, Espagne, Portugal) semblait vouée à l'extinction dans les

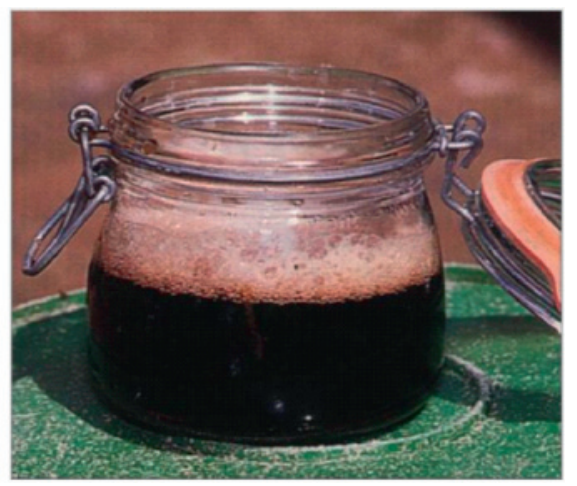

années 1990 (Alzieu et Jacquiet 2011). Mais depuis l'an 2000, sa progression géographique sans précédent en France, a révélé son caractère invasif. Son importance médicale, zootechnique et économique en fait une maladie redoutable, récemment reconnue par l'EFSA (2010) comme une maladie vectorielle émergente des bovins à prendre en considération (Alzieu et Jacquiet 2010, 2011 et 2013, Jacquiet et al 2012b). A l'heure actuelle, de nombreuses zones d'estive pyrénéennes évoluent en état d'endémie. Après les premiers cas recensés dans le début des années 2000, le Massif alpin dans sa partie méridionale et centrale connaît l'installation très préoccupante souvent déjà à l'état endémique de la besnoitiose.

\section{a) Un tableau clinique et lésionnel d'in- tensité croissante}

Les bradyzoïtes des kystes cutanés parasitaires peuvent être transmis mécaniquement d'un bovin infecté à un bovin

Figure 5. La besnoitiose (Besnoitia besnoiti) débute par une phase fébrile (a) suivie d'une phase d'œdèmes ( $b$ et $c$ ) se terminant par une phase de sclérodermie ( $d$ et e) (photos J.P. Alzieu).
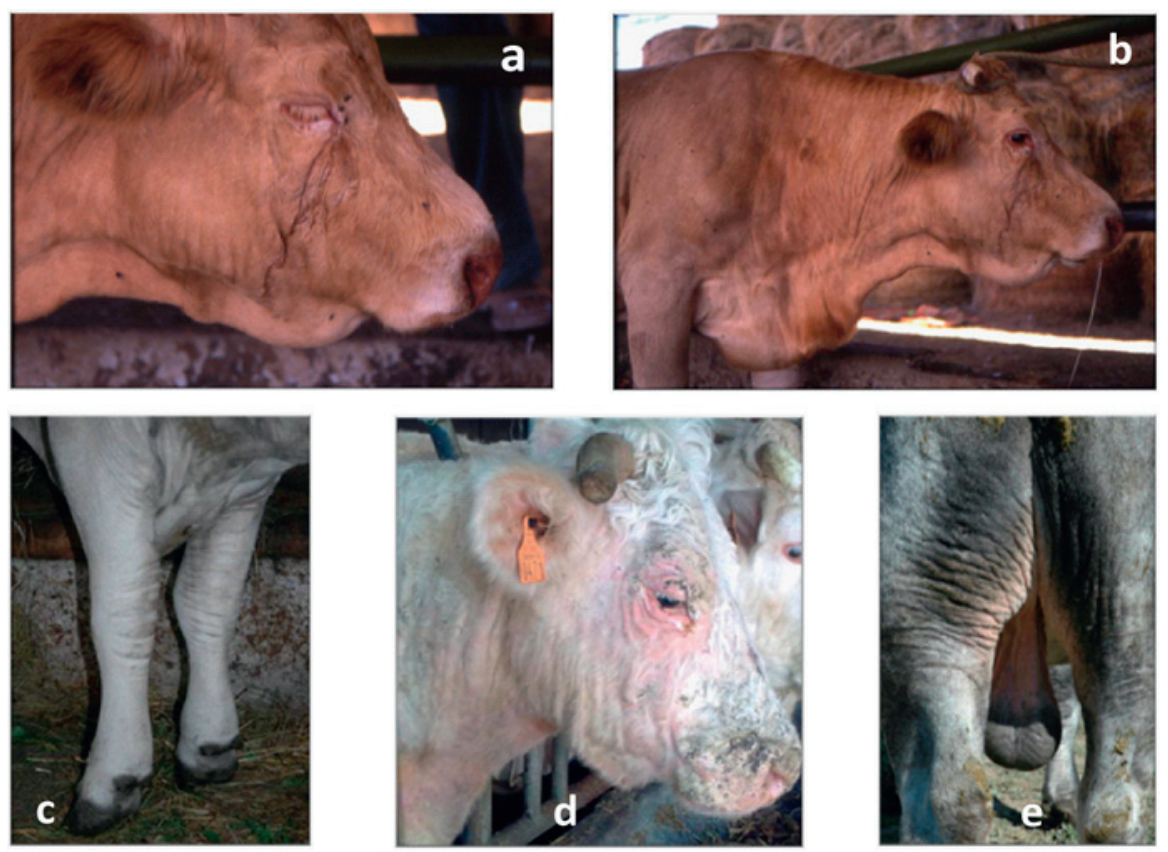
sain par l'intermédiaire d'insectes piqueurs simples vecteurs, principalement des taons et/ou des stomoxes. Ce cycle monoxène de bovin à bovin apparaît désormais dominant.

L'infection par Besnoitia besnoiti est asymptomatique chez 80 à $95 \%$ des sujets contaminés. En revanche, les autres développent dans un délai très variable (de deux semaines à plusieurs mois, un tableau clinique et lésionnel caractéristique, d'intensité croissante au cours du temps. Après une incubation minimale de 6 à 10 jours, trois phases successives peuvent être distinguées (figure 5) : la phase fébrile (durée $=1$ semaine), la phase d'œdèmes (durée $=2$ semaines) suivie d'une phase de plusieurs mois de sclérodermie et dépilations, pouvant conduire à la mort ou à l'euthanasie. Suite à la suspicion clinique, le diagnostic peut être établi de façon formelle par sérologie (Western Blot, ELISA et immuno-fluorescence), à partir de 5 semaines post-infection ou par PCR sur sang et tissu cutané. (Alzieu et Jacquiet 2011, Liénard et al 2012, Schares et al 2013). L'élevage en cheptel collectif avec notamment la pratique de la transhumance estivale, constitue le facteur de risque majeur, tout comme la pratique des achats non contrôlés.

\section{b) Eléments d'épidémiologie}

Sans prédisposition raciale, tous les bovins, quel que soit leur âge, sont réceptifs. Les bovins jeunes apparaissent réceptifs, mais peu sensibles jusqu'à l'âge de un an. Les bovins de 2 à 4 ans et les mâles sont préférentiellement atteints (taux de mortalité plus élevé chez les taureaux) avec une prévalence individuelle de l'ordre de 1 à $3 \%$ en zone d'endémie.

L'estive est particulièrement favorable à la contamination, d'une part, par le mélange de cheptels (la plupart étant composée de sujets infectés) et, d'autre part, par la pullulation estivale de taons et stomoxes, conférant à la maladie une allure pseudo-épizootique. La transmission intra-troupeau est favorisée par l'inféodation des insectes vecteurs à leur troupeau, cherchant toujours à compléter leur repas de sang sur le(s) bovins(s) le(s) plus proche(s) (Alzieu et Jacquiet 2011 et 2012, Liénard et al 2011, Jacquiet et al 2012a, Salem et al 2012).

\section{c) La stratégie de contrôle}

En l'absence de vaccin disponible et devant les résultats souvent médiocres d'un traitement non-stérilisant vis-à-vis du parasite, la stratégie sanitaire doit être adaptée à chaque statut sanitaire. Dans un cheptel infecté, après l'élimination sans délai des malades, et dans

Tableau 2. Les dominantes pathologiques dans l'espèce ovine (par ordre décroissant de fréquence et/ou d'importance).

\begin{tabular}{|l|c|}
\hline Entité pathologique & Importance relative \\
\hline 1. Les plaies & ++++ \\
\hline 2. Les myiases & $++(+)$ \\
\hline $\begin{array}{l}\text { 3. Les boiteries infectieuses } \\
\text { 4. Le poly-parasitisme dont la gale } \\
\text { psoroptique }\end{array}$ & $+++(++)$ \\
\hline $\begin{array}{l}\text { 5. Les troubles respiratoires } \\
\text { infectieux }\end{array}$ & $+(+)$ \\
\hline
\end{tabular}

Légende : + (faible) à ++++ (très élevé), entre parenthèses, les variations possibles.

un second temps, des porteurs de kystes à bradyzoïtes sur les muqueuses, la priorité absolue est d'évaluer la séroprévalence par ELISA sur la totalité du troupeau. Les sujets infectés sont séropositifs de façon pérenne, et donc aisément détectables.

En cas de séroprévalence inférieure à $10 \%$, la prophylaxie sanitaire est conseillée avec la réforme des sujets infectés. Au-delà de $30 \%$, cette réforme devient plus difficile à mettre en œuvre (contraintes économiques fortes) : les cheptels concernés glissent alors de façon inexorable vers l'état d'endémie, avec des pourcentages annuels de nouvelles infections de l'ordre de 30\% (Alzieu et al 2011, Jacquiet et al 2012b).

\section{6 / Le parasitisme interne et externe}

a) Le parasitisme interne

Malgré la «dilution » théorique du réservoir parasitaire dans les pâtures très étendues, le parasitisme interne à nématodes (strongles essentiellement) et à trématodes (fasciolose, paramphistomose) est réellement présent en estive (Mage et Alzieu 1999). Les veaux et bovins de $2^{\text {ème }}$ année de pâture sont infestés par les strongles digestifs, à cycle direct, de la caillette (Ostertagia ostertagi) et de l'intestin grêle.

Contrairement à ce qui a été longtemps prétendu, la limnée tronquée Galba truncatula, hôte intermédiaire de Fasciola hepatica, est présente jusqu'à $2000 \mathrm{~m}$ d'altitude ; elle peut entretenir la contamination par la grande douve le plus souvent, en une seule génération annuelle de mollusques (Mage 1988 et 1989 , Alzieu et Le Goupil 2007). A l'instar de l'espèce ovine (cf. $\S 3.4$ ), si les bovins gagnent l'estive peu parasités, l'estive restera relativement saine; dans le cas inverse, le parasitisme ne fera que s'accroître.

\section{b) Le parasitisme externe}

Il est dominé par l'action perturbante des insectes parasites : les mouches sont responsables de vagues d'allure épizootique de kératoconjonctivites à Moraxella bovis et les tabanidés, de la transmission très efficace de Besnoitia besnoiti. Des cas de myiases sont possibles, mais bien moins prévalents que dans l'espèce ovine (cf. § 3.2) (Alzieu et Gourreau 2005).

\section{3 / Dominantes pathologiques dans l'espèce ovine}

Elles peuvent être classées par ordre décroissant de fréquence et/ou d'importance relative dans l'ordre suivant (qui diffère notoirement de celui des bovins) : les plaies, les myiases, les boiteries, le poly-parasitisme et les troubles respiratoires (Collectif Réseau VEGA 1993a et b), (tableau 2).

\section{1 / Les plaies}

Elles sont particulièrement fréquentes et siègent préférentiellement sur les parties inférieures des membres : leur origine est principalement traumatique (chocs sur les roches, Pyrénées, Alpes, figure 6). Accessoirement des inflammations par piqûres d'insectes (mouches piqueuses, moustiques) créent des plaies sur le corps entier et sur la tête (Collectif Réseau VEGA 1993 b et c).

Si le contrôle médical (soins locaux et/ou généraux) des plaies ne pose guère de problèmes, leur dépistage tardif est source de complications d'abcès, de phlegmons et de myiases. Les ovins s'isolent alors, ne boivent ni ne mangent plus et meurent. Cette situation impose de fait, le regroupement systématique de 
Figure 6. Les plaies chez les ovins sont très fréquentes en estive du fait de la présence de rochers (photo J.P. Alzieu).

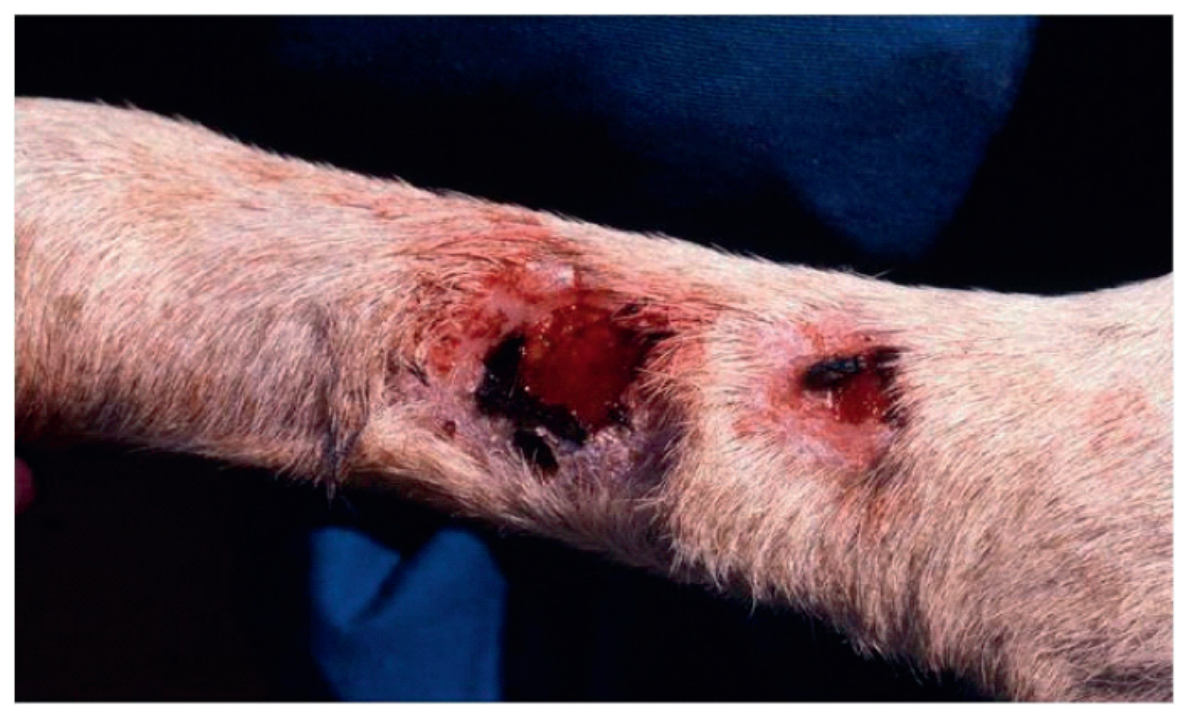

tout le troupeau, au moins une fois par semaine, afin de vérifier l'état clinique de chaque ovin.

\section{2 / Les myiases à Wohlfartia magnifica}

Les myiases primaires sont dues chez les ovins au développement tégumentaire et sous-cutané d'asticots, de larves de certaines mouches et en particulier de Lucilia sericata et de Wohlfartia magnifica (Farkas et Hall 1998, Alzieu et Mage 2004, Alzieu et Gourreau 2005). Cependant, Lucilia sericata ne se développe que dans les zones herbagères françaises de plaines et coteaux et n'est que rarement signalée dans les zones de montagne, toujours à des altitudes inférieures à 800 mètres.

\section{a) Tableau clinique et lésionnel}

A l'inverse de Lucilia sericata (ovipare), Wohlfartia magnifica (mouche larvipare à l'abdomen en damier) semble s'adapter à diverses zones géographiques, mais reste traditionnellement cantonnée, en France, dans les Alpes et dans la partie centrale et occidentale des Pyrénées entre 1000 et 2200 mètres d'altitude (elle est pourtant présente en moyenne altitude en Hongrie et dès 400 mètres en Espagne) (Ruiz-Martinez et al 1991, Hall 1997). La localisation de ces larves est profonde générant des lésions extensives et des galeries (figure 7). Elles sont variablement localisées sur le corps ; la podomyiase est dominante (attirance des mouches par les écoulements de sinus biflexe interdigital) devant les myiases génitales (favorisées par les écoulements naturels du prépuce et de la vulve). A la différence de Lucilia sericata, Wohlfartia magnifica n'a pas besoin de plaies, de tissus lésés ou en décomposition pour se développer. Les surinfections bactériennes locales
$20 \%$ est toujours très supérieure à la mortalité, qui peut quand même selon les années, atteindre et dépasser 3\%. La morbidité est étroitement corrélée à la production et à l'activité des mouches adultes de Wohlfartia, dépendantes des conditions climatiques (elles n'aiment ni le froid, ni le vent, ni la brume). Le développement de Wohlfartia magnifica chez les ovins induit de façon collatérale, l'infestation accidentelle de bovins, équins et caprins (tableau 3 ).

Selon les caractéristiques climatiques et l'orientation des parcours en montagne, la prévalence peut fortement varier. Par exemple, dans les Pyrénées Ariègeoises, la morbidité des ovins a varié de 5 à $10 \%$ dans des endroits géographiquement proches entre 1988 et 1990 . Des enquêtes réalisées dans la même zone ont relevé une morbidité de 3 à $6 \%$ selon les estives lors des étés 2002 et 2003. L'été froid et pluvieux de 2002 a raccourci fortement la saison des myiases (de mi-juillet à mi-août) (Alzieu et Mage 2004, Alzieu et Gourreau 2005). L'évolution des myiases à Wohlfartia en France est habituellement unimodale, avec un pic resserré sur les mois de juillet et d'août.

\section{c) La chimio-prévention}

Le dicyclanil, régulateur de croissance des insectes (Igr IGR), administré par voie cutanée (Alzieu et Gourreau 2005, Sotiraki et al 2005), dès la survenue des premiers cas, en « méta-phylaxie » permet la protection pendant la durée d'activité de Wohlfartia magnifica (généralement de l'ordre de 6 semaines). Le coût du traitement élevé et son application compliquée à moduler dans le temps selon le contexte épidémiologique ont provoqué une certaine désaffection de la part des éleveurs ovins transhumants. depuis 1985 environ. Selon les données européennes, la morbidité variant de 5 à

Figure 7. La mouche Wolhlfartia magnifica est redoutée en estive du fait de l'importance des myiases tégumentaires qu'elle provoque (photo J.P. Alzieu).

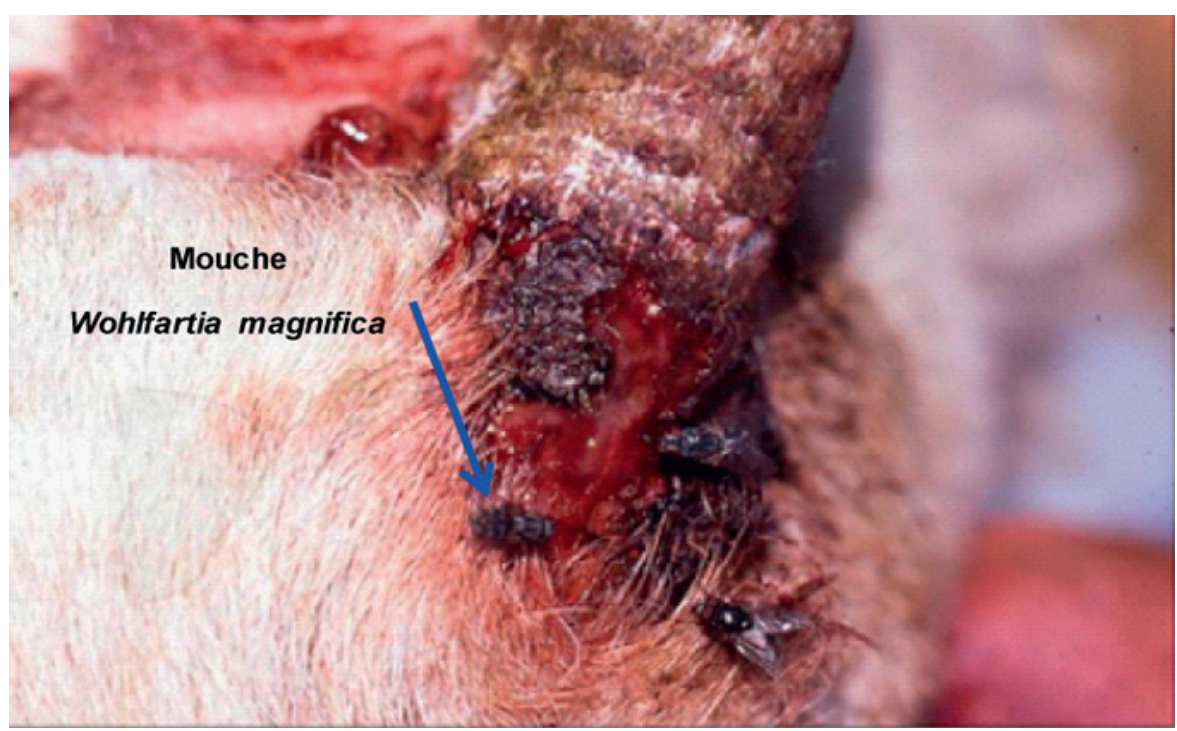


Tableau 3. Principales caractéristiques de la myiase à Wohlfartia magnifica.

\begin{tabular}{|l|l|}
\hline Répartition géographique & $\begin{array}{l}\text { Altitude (> 800 m) } \\
\text { Surtout dans les massifs montagneux } \\
\text { (Alpes et Pyrénées). }\end{array}$ \\
\hline Répartition biologique & $\begin{array}{l}\text { Larvipare } \\
\text { Localisation profonde des asticots }\end{array}$ \\
\hline \multirow{2}{*}{ Espèces affectées } & $\begin{array}{l}\text { Ovins, caprins, chameaux, chevaux, ânes, } \\
\text { bovins, (chiens, volailles), } \\
\text { (quelques cas seulement chez l'Homme). }\end{array}$ \\
\hline Période d'infestation & De juillet à septembre \\
\hline \multirow{5}{*}{ Contexte tégumentaire } & $\begin{array}{l}\text { Tissus sains ou plaies } \\
\text { Orifices naturels } \\
\text { Espace inter-digité }\end{array}$ \\
\hline & $\begin{array}{l}\text { Espace inter-digité } \\
\text { Oreilles } \\
\text { Peau et toison } \\
\text { Tronc et arrière-train } \\
\text { Vulve ou fourreau }\end{array}$ \\
& +++ \\
& $++(+)$ \\
\hline
\end{tabular}

Légende : + (faible) à ++++ (très élevé), entre parenthèses, les variations possibles.

\section{3 / Les boiteries infectieuses}

Les boiteries infectieuses sont de deux ordres ; celles accidentelles, en relation avec le phlegmon inter-digité et celles, beaucoup plus fréquentes résultant de complications d'un fourchet ou de piétin préexistant (Collectif Réseau VEGA 1993 b et c).

\section{a) Le phlegmon inter-digité}

Les symptômes, les conditions de survenue et les possibilités de contrôle sont identiques à celles observées chez l'espèce bovine (cf. § 2.2.a) : le début de la période d'estive avec une humidité supérieure semble plus favorable à la survenue du phlegmon inter-digité. Son incidence décroît ensuite en fin de période d'estive. Son incidence cumulée est très variable, mais est habituellement voisine de $5 \%$.

b) Les complications des boiteries antérieures : fourchet et piétin

Le fourchet (ou dermatite inter-digitée) est dû au germe de la nécrose Fusobacterium necrophorum et est qualifié souvent de " piétin bénin », lié à des blessures ou à des macérations locales. Le piétin, beaucoup plus grave, est dû à l'action synergique de $F$. necrophorum et de Dichelobacter nodosus (d'origine digestive) : cette dernière bactérie est peu résistante dans le milieu extérieur, mais persiste au niveau du pied, par son action protéolytique et son pouvoir d'altération de la corne.

Des suivis épidémiologiques sur les estives pyrénéennes ont révélé que le piétin d'évolution enzootique était la cause d'environ deux tiers des boiteries. Son taux d'incidence cumulée atteignait dans ces suivis, près de $14 \%$ : cela constitue donc une charge supplémentaire de travail notoire pour les bergers et une source de frais vétérinaires supplémentaires (Collectif Réseau VEGA 1993b et c). Il a été démontré que les ovins gagnant l'estive boiteux (généralement non guéris et sources d'infection) redescendent de l'estive boiteux : au lieu d'être un facteur de guérison par la dilution du microbisme, l'estive maintient, voire même accroît, la source infectieuse pour le troupeau.

Le contrôle du piétin est connu et bien documenté (parage, traitement local, pédiluve, anti-infectieux et vaccination éventuelle). Le travail de prévention doit donc être clairement réalisé avant l'estive, en bergerie : la plupart des éleveurs le font (parage des onglons), mais paient parfois pour la frange de ceux qui de la laine (b) chez la brebis (photos J.P. Alzieu).

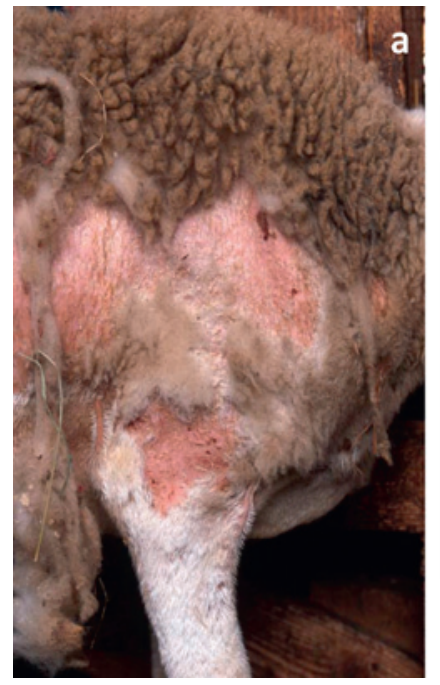

ne le font pas (archétype d'une dominante pathologique qui pourrait ne pas l'être).

\section{4 / Le parasitisme interne et externe}

a) Le poly-parasitisme interne

Les ovins sont traditionnellement sujets à un poly-parasitisme interne assez intense, dominé par la monieziose et les strongyloses digestives chez les agneaux et agnelles, et par les strongyloses digestives et autres parasitoses à cycle indirect (protostrongylinoses, dicrocoeliose, fasciolose).

L'estive pourrait constituer un facteur de dilution du parasitisme par la surface de pâture disponible. En effet, la contamination résiduelle du pâturage est faible en sortie de l'hiver. Hélas, dans de nombreux cas, les troupeaux constituant le cheptel collectif maîtrisent de façon imparfaite ce parasitisme (traitements de routine sans contrôle régulier par coproscopie du parasitisme présent). De ce fait, il y a fort souvent recyclage et amplification du parasitisme en estive et à l'instar des boiteries infectieuses, les troupeaux peuvent redescendre de l'estive encore plus parasités (résultats d'enquêtes épidémiologiques pyrénéennes) (Collectif Réseau VEGA 1993d).

b) Le parasitisme externe dominé par la gale psoroptique

Mis à part le contexte sérieux des myiases à Wohlfartia (cf. § 3.2), le parasitisme externe des ovins est dominé par la très forte prévalence de la gale psoroptique ovine, due à l'acarien Psoroptes ovis, quasi-généralisée aux cheptels collectifs des massifs montagneux (Collectif Réseau VEGA 1993b et c). Cette dermatose est très contagieuse et génère

Figure 8. La gale psoroptique provoque des lésions d'hyperkératose (a) et une perte

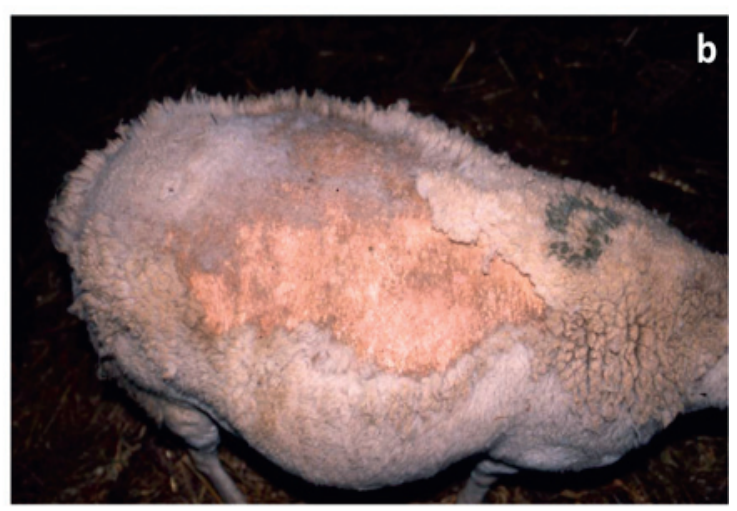


prurit, dépilations (perte de laine) avec hyperkératose (figure 8), en particulier en phase hivernale en bergerie. A la montée en estive, une fois les ovins tondus (et variablement traités), la gale se manifeste peu, en dépit de la persistance des psoroptes dans des zones-refuges (entrée de l'oreille, coin des yeux, fosses ischiales) : la gale du corps s'avère donc peu visible en estive.

Pourtant, les contacts entre ovins, accrus lors des rassemblements pour soins et observations du troupeau se multiplient et permettent l'infestation à bas bruit de l'ensemble des cheptels du troupeau collectif, asymptomatique jusqu'à l'entrée de l'hiver. C'est ainsi que se maintient le véritable fléau de la gale psoroptique dans les cheptels alpins et pyrénéens, sans pression règlementaire depuis son abolition de la liste des maladies légalement contagieuses. Son contrôle est pourtant possible par des traitements méta-phylactiques avec des endectocides (lactones macrocycliques) appliqués stratégiquement dès la descente et avant la montée en estive : un certain nombre d'éleveurs réalisent correctement ces traitements, les autres continuant à entretenir la maladie, ce qui est difficile à vivre pour ceux qui visent l'éradication de la gale.

\section{5 / Les troubles respiratoires infectieux}

Les ovins apparaissent beaucoup moins sensibles que les bovins. Toutefois, à la montée en estive, si le temps est très froid ou présente de fortes et brutales variations climatiques, des épisodes graves, d'allure quasi-épizootique, de congestion active pulmonaire peuvent survenir (la température critique inférieure de l'ovin tondu devenant $20^{\circ} \mathrm{C}$ !). Des épisodes avec une mortalité de l'ordre de 10 à $20 \%$ ont été décrits dans les Pyrénées (observations personnelles J.P. Alzieu) avec des surinfections bactériennes principalement par Pasteurella multocida $s p$.

\section{Conclusion}

Mis à part certaines pathologies propres à l'estive comme la myiase à Wohlfartia magnifica, la plupart des pathologies dominantes sont importées sur les esti- ves avec leurs hôtes bovins et ovins. La problématique des cheptels collectifs ne fait qu'accroître les risques sanitaires (la besnoitiose en est sans doute le meilleur exemple). La rudesse du climat et ses fréquentes variations thermiques, constituent des facteurs hautement favorisants pour les maladies respiratoires en particulier dans l'espèce bovine.

Plusieurs études d'épidémio-surveillance réalisées entre 1990 et 2000 avaient déjà montré (Collectif Réseau VEGA 1993a, b et d), la nécessité d'avoir dès la montée en estive, un état sanitaire optimal et ce quelle que soit l'espèce. Les cheptels " sains » dans une estive bien gérée redescendent en bon état corporel et sanitaire. A contrario, les cheptels en état sanitaire médiocre ne peuvent compenser par le seul fait d'être montés en estive leur handicap et paient le plus lourd tribut aux pathologies. La fréquence des troubles observés, d'un avis général encore trop élevée, souligne tous les efforts de prévention nécessaires à accomplir dans les élevages d'origine.

La vie et l'élevage en montagne n'en seraient que plus idylliques! Utopie?

\section{Références}

Alzieu J.P., 2007. Reemerging cattle besnoitiosis (Besnoitia besnoiti) in France: update on clinical and epidemiological aspects. Proc. WAAVP Congr., Ghent.

Alzieu J.P., Chartier C., 2007. La coccidiose bovine : actualités épidémiologiques, diagnostiques et thérapeutiques. Bull. GTV, Hors-série, Parasitologie des bovins, 89-95.

Alzieu J.P., Dorchies P., 2007. Le parasitisme interne du jeune cheval entre sevrage et débourrage. Journ. Nat. GTV, Nantes, France, 255-270.

Alzieu J.P., Gourreau J.M., 2005. Actualités sur les myiases externes ovines. Le Point Vétérinaire, 256, 22-28.

Alzieu J.P., Jacquiet P., 2012. La besnoitiose bovine : du constat de son émergence à la nécessité de son contrôle. Le Point Vétérinaire, $\mathrm{N}^{\circ}$ Spécial Parasitologie interne des ruminants, 106-112.

Alzieu J.P., Le Goupil V., 2006. Comprendre la recrudescence de la coccidiose bovine : actualités épidémiologiques et facteurs de risque. Journ. Nat. GTV, Dijon, France, 367-373.

Alzieu J.P., Le Goupil V., 2007. La fasciolose bovine : toujours présente, une gravité sournoise. Bull. GTV, Hors-série Parasitologie des bovins, 75-82.

Alzieu J.P., Mage C., 2004. Actualités en entomologie : myiases et mouches. Conséquences et stratégies thérapeutiques. Journ. Nat. GTV, Tours, France, 589-600.

Alzieu J.P., Jacquiet P., Liénard E., Malavieille R., Grisez C., Prévot F., Bergeaud J.P., Desclaux X., Dorchies P., Franc M., 2010. L'inexorable progression de la besnoitiose bovine en France : actualités cliniques, épidé- miologiques et diagnostiques. Journ. Nat. GTV, Lille, France, 921-926.

Alzieu J.P., Jacquiet P., Liénard E., Franc M., 2011. New data on kinetics of infection by Besnoitiosis in cattle herds from endemic and non-endemic French areas Proc. EBF 2011, Marseille, 16-18 Nov. p25.

Alzieu J.P., Jacquiet P., Liénard E., Grisez C., Prévot F., Malavieille R., Desclaux X., Nussbaum S., Bergeaud J.P., Franc M., Schelcher, F., Corboz, N., Bastien, F., GuerrierChatelet M.C., Gavet L., Schneider V., Boulon C., 2011. Réémergence de la besnoitiose bovine : démarche diagnostique et possibilités de contrôle. Bull. GTV, 71-86.

Alzieu J.P., Dorchies P., Goyeau K., Ferrier T., Bonadei M., Rameil M., Desclaux X., Malavieille R., Nussbaum S., 2012. Actualités sur l'épidémiologie de la coccidiose bovine : résultats d'un suivi multicentrique français. Journ. Nat. GTV, Nantes, France, 541-552.

Chauvin A., Malandrin L., Bonnet S., L'Hostis M., 2007. Diagnostic et thérapeutique des babésioses chez les bovins. Le Nouveau Praticien Vétérinaire : élevages et santé, 6, 28-34.

Collectif-Réseau VEGA, 1993a. Surveillance épidémiologique des estives plates. Réseau d'Epidémio-surveillance, VEGA, $4^{\text {ème }}$ trimestre, 4, 4-7.

Collectif-Réseau VEGA, 1993b. Résultats généraux des enquêtes ovine et bovine. Réseau d'Epidémio-surveillance, VEGA, $4^{\text {ème }}$ trimestre, 4, 8-10.

Collectif-Réseau VEGA, 1993c. Etude clinique d'une estive ovine exemplaire. Réseau d'Epidémio-surveillance, VEGA, $4^{\text {ème }}$ trimestre, 4, 11-14.
Collectif-Réseau VEGA, 1993d. Etude du parasitisme ovin sur les estives : l'infestation parasitaire des ovins à la descente est fortement conditionnée par leur statut parasitaire à la montée. Réseau d'Epidémio-surveillance, VEGA, $4^{\text {eme }}$ trimestre, 4, 15-16.

Collectif-Réseau VEGA, 1993e. Résultats généraux de la surveillance clinique bovine. Réseau d'Epidémio-surveillance, VEGA, $4^{\text {ème }}$ trimestre, 4, 17-19.

Devos J., Geysen D., 2005a. La babesiose bovine à Babesia divergens. Bulletin des GTV, 31, 55-59.

Devos J., Geysen D., 2005b. Etude épidémiologique : la babésiose bovine : enquête dans une clientèle vétérinaire des Monts du Lyonnais. Bull. GTV, 31, 61-67.

Dorchies P., 2007. Réchauffement climatique et parasitisme. Bull. GTV, Hors-série, Parasitologie des bovins, 15-22.

Dorchies P., Alzieu J.P., 2008. L'épidémiologie, clé de la connaissance de la fasciolose bovine : l'exemple de la France. Du dépistage au contrôle : le rôle essentiel du vétérinaire. 4887 in Collectif « La fasciolose bovine », Virbac Ed., 144p.

Farkas R., Hall M.J., 1998. Prevalence of traumatic myiasis in Hungary: a questionnaire survey of veterinarians. Vet. Rec., 143, 440443.

Hall M.J., 1997. Traumatic myiasis of sheep in Europe: a review. Parasitologia. 39, 409-413.

Jacquiet Ph., Grisez C., Chauvin A., Prévot F., Liénard E., Bergeaud J.P., Dorchies P., Alzieu J.P., 2010. Absence d'hypobiose hivernale chez les nématodes parasites de la caillette des bovins 
dans le Sud-Ouest de la France. Journ. Nat. GTV, Lille, France, 927-936.

Jacquiet Ph., Alzieu J.P., Liénard E., Grisez C., Prévot F., Malavielle R., Desclaux X., Nussbaum S., Bergeaud J.P., Franc M., Boulon C., Méjean F., 2012a. Dynamique de la transaction de la besnoitiose bovine à Besnoitia besnoiti entre troupeaux et au sein de troupeaux infectés. Journ. Nat. GTV, Nantes, France, 535540 .

Jacquiet P., Alzieu J.P., Liénard E., Prévot F., Grisez C., Boulon C., Franc M., 2012b. Is it possible to stop the spread of bovine besnoitiosis in areas of emergence ? ApiCOWplexa, Apicomplexa in farm animals. Int. Meet. Lisbon, Portugal, 25-28 October, Proc., p8.

L'Hostis M., Chauvet S., Devers P., Chauvin A., Tiques et babésioses : transmission et aspects cliniques chez les bovins. Le Nouveau Praticien Vétérinaire : élevage et santé, 6, 2126.

Liénard E., Alzieu J.P., Prévot F., Grisez C., Malavieille R., Desclaux X., Salem A., Bergeaud J.P., Franc M., Jacquiet P., 2011. Dynamics of bovine Besnoitia besnoiti (Apicomplexa, Sarcocystidae) infections: within and between farms spreads. XXIII Int. Conf. WAAVP, Buenos Aires, Argentina.
Liénard E., Alzieu J.P., Grisez C., Prévot F., Bardoux P., Blanchard B., Salem A., Franc M. Jacquiet P., 2012. Blurred epidemiology of bovine besnoitiosis: parasite detection in skin among seropositive cattle. ApiCOWplexa. Apicomplexa in farm animals. Int. Meet. Lisbon, Portugal, Poster 57, p122.

Mage C., 1988. Contribution à l'étude de la fasciolose à Fasciola hepatica $L$. des bovins allaitants dans le Limousin et la Cerdagne (France). Conséquences zootechniques et essais thérapeutiques. Thèse Doctorat d'Université de Limoges, 136p.

Mage C., 1989. Epidémiologie de Fasciola hepatica chez les jeunes bovins élevés dans les alpages de la Cerdagne. Rev. Méd. Vét., 140, 1033-1036.

Mage C., Alzieu J.P., 1999. Parasitisme, système d'élevage et extensification. Quelle stratégie pour demain? Comprendre la clinique pour une prescription appropriée. Journ. Nat. GTV, INRA, Nantes, France, 271-280.

Memeteau S., 2011. Situation épidémiologique actuelle de la rhinotrachéite infectieuse bovine en France chez les bovins. Le Nouveau Praticien Vétérinaire, 19, 25-28.

Ruiz Martinez I., Soler Cruz M.D., Benitez Rodriguez R., Perez Jimenez J.M., Diaz Lopez
M., 1991. Myiasis caused by Wohlfahrtia magnifica in sheep and goats in southern Spain II. Effect of age, body region and sex on larval infestation. Israel J. Vet. Med., 46, 64-68.

Salem A., Franc M., Jacquet P., Bouhsira E., Liénard E., 2012. Feeding and breeding aspects of Stomoxys calcitrans (Diptera: Muscidae) under laboratory conditions. Parasite, 19, 309317.

Schares G., Langenmayer M.C., Scharr J.C., Minke L., Maksimov P., Maksimov A., Schares S., Bärwald A., Basso W., Dubey J.P., Conraths F.J., Gollnick N.S., 2013. Novel tools for the diagnosis and differentiation of acute and chronic bovine besnoitiosis. Int. J. Parasitol., 43, 143-54.

Schelcher F., Foucras G., Corbière F., Maillard R., Raboisson D., Meyer G., 2011. IBR chez les bovins : de la latence à la vaccination. Le Nouveau Praticien Vétérinaire, 165, 14-24.

Sotiraki S., Stefanakis A., Hall M.J.R., Graf J.F., 2005. Field trial of the efficacy of dicyclanil for the prevention of wohlfartiosis of sheep. Vet. Rec., 156, 37-40.

\title{
Résumé
}

Mis à part certaines pathologies spécifiques telle la myiase à Wohlfartia magnifica, la plupart des maladies infectieuses et parasitaires d'importance sont véhiculées sur l'estive avec leurs hôtes, bovins et ovins. La problématique des cheptels collectifs constitués de troupeaux de statuts sanitaires différents et les fortes variations climatiques et thermiques favorisent l'expression des maladies infectieuses. La montée en estive d'animaux infectés constitue le facteur majeur de risque, induisant à terme un accroissement de la morbidité, de la mortalité et des pertes économiques. La meilleure prévention réside dans la meilleure maîtrise sanitaire des troupeaux avant leur transhumance.

\begin{abstract}
Pathological features of domestic ruminants in mountain pastures in France

Except some specific diseases such as Wohlfartia magnifica myiasis, most of the main infectious and parasitic diseases occurring on mountain pastures result from a host carriage of pathogens. Mixed herds with their own sanitary status and huge climatic and thermal variations favour infectious disease outbreaks. Transhumance with infected carriers is the major risk factor inducing higher morbidity, mortality and economic losses during summering on mountain pastures. The best solution remains the preventive sanitary management of the herds before this transhumance.
\end{abstract}

ALZIEU J.-P., BRUGÈRE-PICOUX J., BRARD C., 2014. Particularités pathologiques des ruminants domestiques en estive dans les montagnes françaises. In : Spécificités de l'élevage de ruminants en montagne. Grosclaude J., Thibier M., Baumont R. (Eds). Dossier, INRA Prod. Anim., 27, 31-40. 